\title{
Dichoptic metacontrast masking reveals a central basis for monoptic chromatic induction
}

\author{
CONRAD X. OLSON and ROBERT M. BOYNTON \\ University of California at San Diego, La Jolla, California
}

\begin{abstract}
Using a magnitude estimation method, an assessment was made of the nature and strength of the hue induced into an initially white test field by an adjacent chromatic inducing field dolivered to the same eye. On half the trials, a metacontrast mask, seen as flanking the inducing field but delivered to the other eye, eliminated the perception of the inducing field without having any direct effect upon the appearance of the test. Strength of induction with the mask was only a small fraction of its unmasked value. Because the mask could not have affected any retinal component of induction, it is concluded that the basis of the chromatic induction is largely or entirely nonretinal.
\end{abstract}

Researchers have long considered chromatic induction to be a phenomenon resulting primarily from neural interactions in the retina with little processing occurring more centrally. There have been some exceptions to this view: Parsons (1924) suggested that weak color contrast effects may be achieved haploscopically. The colors seen by binocular combination of two-primary-color photographs (Geschwind \& Segal, 1960) provide justification for implicating central structures in the processing of chromatic induction. See Graham and Brown (1965, pp. 452-478) for further discussion.

Helmholtz (1866/1924) invoked a high-level mechanism involving "errors of judgment" and "unconscious inferences," implying that color induction is an effect influenced not only by perceptual signals, but also by coded experience. Hering (1890) countered convincingly that color induction could be explained without resorting to explanations implicating cognitive processing. Strong support for his thesis came from the results of an experiment in which two backgrounds, red and blue, were presented haploscopically to form a single, homogeneous violet field. Hering placed a gray field on each background, vertically displaced to avoid binocular combination. Although Helmholtz's theory would predict that each gray patch should appear as a greenish yellow, Hering found that the gray patch on the red background appeared green and on the blue background appeared yellow. Because of this demonstration of monocular

This research was supported by Grant EY-01451 from the National Eye Institute, US Public Health Service. Please address all correspondence to Robert M. Boynton, Psychology C-009, University of California at San Diego, La Jolla, CA 92093. A preliminary report of this experiment, based on data from only one observer, was included as part of a paper read at the NATO Colour Vision Conference at Trinity College, University of Cambridge, in August 1982 (Mollen \& Sharpe, 1983). The authors wish to express their appreciation to Allen L. Nagy (who also served as the third subject), and to D. I. A. MacLeod, Melissa L. Monty, and D. Leo Stefurak for discussions related to this work. independence, Hering concluded that no. higher level structures mediate chromatic induction. His experiment does not, however, logically rule out the possibility of lateral interactions between signals in the monocular visual pathways of the brain.

In 1966, Wiesel and Hubel reported the discovery of single-opponent cells in the primate retina. These so-called Type 1 cells, which have been consistently observed by many other investigators since their initial discovery, have red on-centers and green offsurrounds, or green on-centers and red off-surrounds. They constitute most of the opponent-color cells found in the macaque retina. It is clear that these cells cannot account for chromatic induction. What is needed instead are double-opponent cells, with redon, green-off centers and green-on, red-off surrounds (or the converse). However, despite nearly two decades of searching, no double-opponent cells have been found in the primate retina.

Despite this lack of physiological evidence, the tacit assumption that the retina alone processes color induction has persisted among many recent researchers, including Alpern (1964), DeValois and Walraven (1967), Jameson and Hurvich (1961, 1964), Hurvich (1981), Kinney (1962, 1967), Walraven (1981), and Wooten (1970).

With the discovery of monocularly driven, dualopponent color receptive cells in the monkey striate cortex (Michael, 1978a, 1978b, 1978c), there is physiological evidence that a mechanism for chromatic induction exists at the cortical level. Consistent with these electrophysiological data, we present psychophysical evidence that, although confined to monocular visual pathways, the processing of signals underlying chromatic induction is primarily nonretinal.

\section{METHOD}

\author{
Apparatus \\ Using the La Jolla Analytic Colorimeter (Boynton \& Nagy, \\ $1981,1982)$, two rectangular fields, arbitrarily named inducing
}


(I) and test ( $\mathrm{T})$, were presented to the right eye. The two fields were initially white, each produced by an identical physical mixture of four nearly monochromatic primaries $(437,488,563$, and $655 \mathrm{~nm}$ ). The 437 - and $488-\mathrm{nm}$ primaries were never varied during the experiment. The chromaticity of either field could be varied by exchanging the proportions of the 563 - and $655-\mathrm{nm}$ components, causing the excitation of the long-wavelength-sensitive (R) cones either to increase or decrease while producing an opposite change of middle-wavelength-sensitive $(G)$ cone excitation calculated to keep luminance constant at $0.16 \mathrm{~cd} / \mathrm{m}^{2}$.

The spatial and temporal conditions of the experiment are shown in Figure 1. All stimulus fields were provided by rear projection. A hexagonal fixation array of luminous dots, fused haploscopically, surrounded the fields. Two square masking fields, presented only to the left eye, and seen as flanking the inducing field, were about equal in luminance but slightly more reddish in appearance than the test and inducing fields. Head position was fixed using the bite-bar method. All manipulations of the stimuli were produced using digital electronic equipment.

\section{Conditions}

A-No mask. Designed as a control, this condition consisted of presentation of I and T only, each for $100 \mathrm{msec}$, every $20 \mathrm{sec}$. The onset of I preceded that of $\mathrm{T}$ by $50 \mathrm{msec}$. Within the no-mask condition, the chromaticity of either I or $T$ was manipulated, while the other field remained unchanged at the white neutral point. The manipulated field could be set to one of nine different chromaticities, ranging from a fairly saturated green through neutral to a fairly saturated red. The expected results were that: (1) when the varied field appeared reddish, it would induce a greenish color into the unvaried field, and vice versa; and (2) the saturation of the induced color would vary directly with that of the inducing color.

B-Mask. Condition B was in all respects identical to Condition $A$, except that a metacontrast mask, $M$, delivered only to the left eye, was perceived as flanking the inducing field. The metacontrast mask was chosen for its ability to suppress the perception of I dichoptically. Any visual phenomenon interrupted by such a mask must be processed beyond the level of binocular combination of visual signals (Werner, 1940). The suppression of the perception

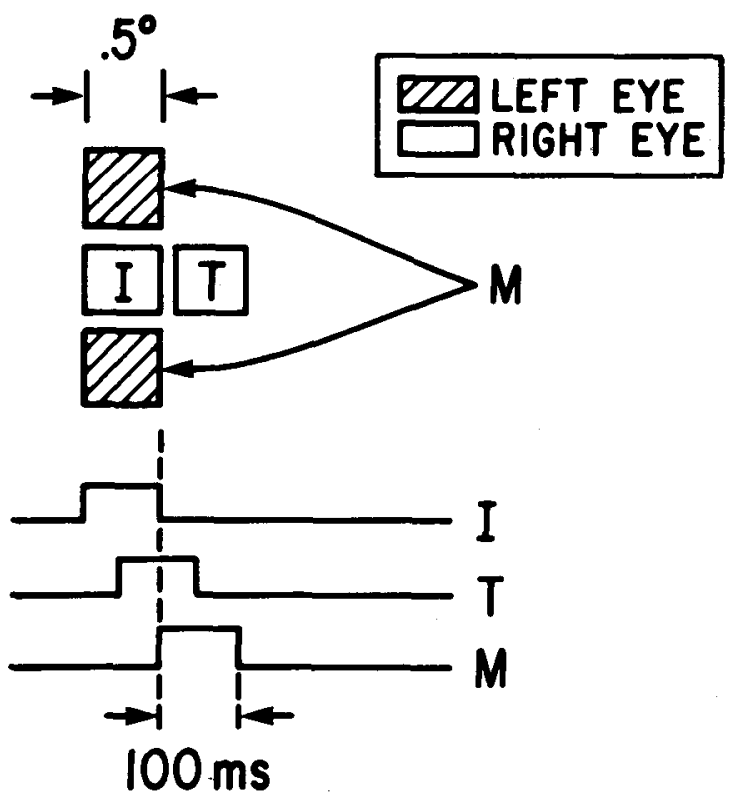

Figure 1. Spatial and temporal arrangement of the test (T), inducing (I), and metacontrast masking (M) stimuli of the experiment. of a visual stimulus through metacontrast tends to be global, despite the spatial and temporal specificity of the conditions that produce it. Alpern $(1953,1954)$ and others have shown that the smaller the visual angle between target and mask, the greater is the effect, with metacontrast masking best at zero distance, weak at $1 \mathrm{deg}$, and eliminated at $2 \mathrm{deg}$ of visual angle. Maximum suppression of the inhibited stimulus is achieved at about $80-100 \mathrm{msec}$ SOA, with the mask following the stimulus to be suppressed. The effectiveness of the mask is greatly reduced both before and after this temporal displacement. For reviews, see Lefton (1973) and Uttal (1981, pp. 902-913).

The spatial and temporal configurations were intended to provide maximal suppression of the inducing field with minimal direct influence on the perception of the test. The onset of $M$ followed that of I by $100 \mathrm{msec}$, and each lasted $100 \mathrm{msec}$. The metacontrast mask thereby eliminated the perception of the inducing field without removing its physical presence.

\section{Procedures}

Conditions A and B were run together. Within each condition, either I or $\mathrm{T}$ remained white while the chromaticity of the other field was set at one of nine values. These included the matching white and four levels of red or green produced by either increasing or decreasing the degree of R-cone excitation by exchange of the 563 - and $655-\mathrm{nm}$ primaries. This yielded a total of 17 possible stimuli (the condition in which both fields were unchanged was not duplicated). Thus, there were 34 possible stimuli in the two conditions. Their ordering was randomized, run forward and backward, and rerandomized; this was done four more times to produce 10 presentations of each stimulus. Whether Condition $A$ or Condition B would occur on a particular trial was also randomly determined.

Subjects were instructed to estimate the magnitude of saturation of the test field on the following scale: $G 3$, strong green; $G 2$, moderate green; G1, weak green; N, neutral; R1, weak red; R2, moderate red; R3, strong red. In Condition $B$, the subject was also instructed to judge whether he saw the inducing field, using the following scale: 0 , definitely no; 1 , probably no; 2 , probably yes; 3, definitely yes. The experimenter and subject, who were in separate rooms, communicated electronically. The subject reported his judgment after each stimulus presentation. Soundproofing precluded auditory cues.

\section{Subjects}

Although we tried this experiment on five subjects, we were successful with only three of them. For the other two, both of whom exhibited chromatic induction when 1,000-msec flashes were used, it proved impossible, with 100 -msec flashes, to produce significant chromatic induction; metacontrast is not effective when longer flashes are used. The ages of the three subjects, all experienced observers with normal color vision and spatial acuity (corrected for RMB, a presbyope), were 20 (C.X.O.), 35 (A.L.N.), and 58 (R.M.B.) years.

\section{RESULTS}

\section{Scoring System}

Responses of "red" were designated as positive, and "green," negative, weighted according to the point values assigned to the subject's response. If a subject were to judge a particular stimulus as "strong red" on each of 10 experimental trials, his magnitude estimation score would be $+3 \times 10=+30$. For "strong green" on all 10 trials, the score would be $-3 \times$ $10=-30$. A score of zero could result, for example, from seven neutral, one R3, and three $\mathrm{G} 1$ responses. The slope of the regression line is arbitrarily defined 
so that it would be unity if the line extended from +30 to -30 for the full extent of change in the $R-$ cone excitation used $\pm 10 \%$. The lines fitted to the points representing judgments of $T$ when its color resulted from the action of $I$ have slopes $m_{i}$ ( $i$ for induced), which are generally negative, indicating that a change in I toward red produces a judgment of green in $T$, and vice versa. The lines fitted to points representing judgments of $T$ when $T$ itself was varied have positive slopes $m_{d}(d$ for direct). The magnitude of induction is measured by taking the ratio $-m_{i} / m_{d}$ and representing the result as a percentage. Thus, $50 \%$ induction means that the slope of the function that gauges the opponent hue caused by induction is half that of the hue change produced by an equivalent change in the chromaticity of the test field itself.

\section{No-Mask Results}

For stimuli presented without the metacontrast mask, mean data for three subjects are shown in Figure 2. In this figure, the abscissa represents the percent change in R-cone excitation, and the ordinate represents the mean of the summed ratings assigned to the hue of the test field in 10 experimental trials. The mean data for the three subjects show $m_{i}=-.35$ and $\mathrm{m}_{\mathrm{d}}=.72$ (49\% chromatic induction). Individual data are shown as regression lines in the top half of Fig-

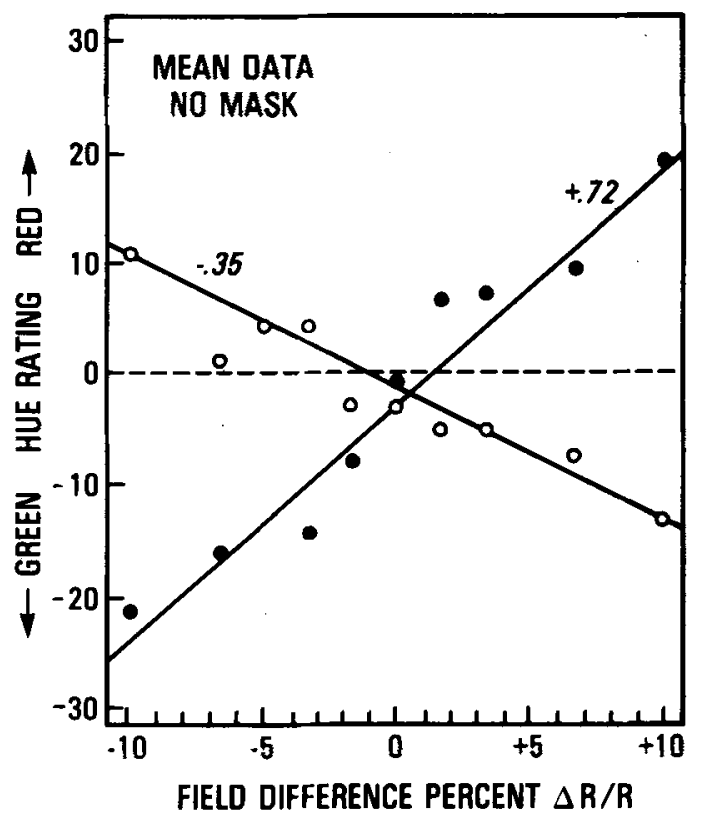

Figure 2. Mean data of the three subjects of the experiment for Condition A (no mask). The ordinate shows the mean of the summed hue ratings for each subject. The abscissa indicates the chromaticity change of either $T$ or I specified in terms of a calculated percent difference in R-cone excitation, assuming the Smith and Pokorny (1975) cone action spectra. The slopes of the regression lines that provide the optimal least-squares fit to the data points (filled circles for the direct effect, open circles for the induced effect) are shown. ure 3, where slopes can be estimated visually. The figure legend gives coefficients of correlation to indicate the degree to which the plotted lines describe the data points, which, to avoid crowding, have been left off the figure.

\section{Results With the Mask}

In preliminary experiments, Subjects R.M.B. and C.X.O. reported seeing the inducing field on occasion despite the mask. Therefore, they were asked to judge not only the magnitude of the hue in $T$, but also whether or not they saw I. C.X.O. reported that he "probably" saw I in fewer than $2 \%$ of the trials; he reported no cases in which he "definitely" saw it. R.M.B. reported that he "probably" saw I in just over $1 \%$ of the trials; he reported that he "definitely" saw it in an additional $0.6 \%$ of the trials. Most such trials occurred when I was strongly chromatic, consistent with the results of Yellott and Wandell (1976), who studied metacontrast as a function of the hue difference between mask and test. The metacontrast mask was extremely effective for Subject A.L.N., who never reported seeing the inducing field.

Figure 3 (bottom) and Figure 4 show the results for Condition B. The elimination of the perception of the inducing field, though not its physical presence, also eliminated most, and perhaps all, of the chromatic induction. For each subject, the slope $m_{d}$ of the line representing the estimate of the hue change when $T$ itself was varied was not materially altered by the mask, which indicates that the presence of the mask did not affect the magnitude estimation of the hue of the test field. However, the slope $m_{i}$ of the induced effect was greatly reduced in each case (R.M.B., 0.52 to 0.13 ; A.L.N., 0.48 to -0.19 ; C.X.O., 0.38 to 0.04). The mean data for the three subjects shown in Figure 4 yield only a $2 \%$ induction effect, suggesting that the induction in the presence of the mask is either insignificant or eliminated completely.

\section{DISCUSSION}

The results indicate that the suppression of perception of the inducing field, at a level of visual processing at or beyond that where signals from the two eyes are initially combined, greatly reduced or eliminated chromatic induction. The masking fields did not interrupt or alter the subjects' judgments of the test field when $T$ was varied. Nor could the retinal encoding of the inducing field have been blocked by the mask delivered to the contralateral eye.

These findings are of inierest relative to the electrophysiological data of Michael (1978a, 1978b, 1978c), who reports the presence of double-opponent cells in the striate cortex of the rhesus monkey. These cells, which Michael says are exclusively monocular, form the basis for a physiological model of chromatic induction in which receptive fields in the retina, con- 


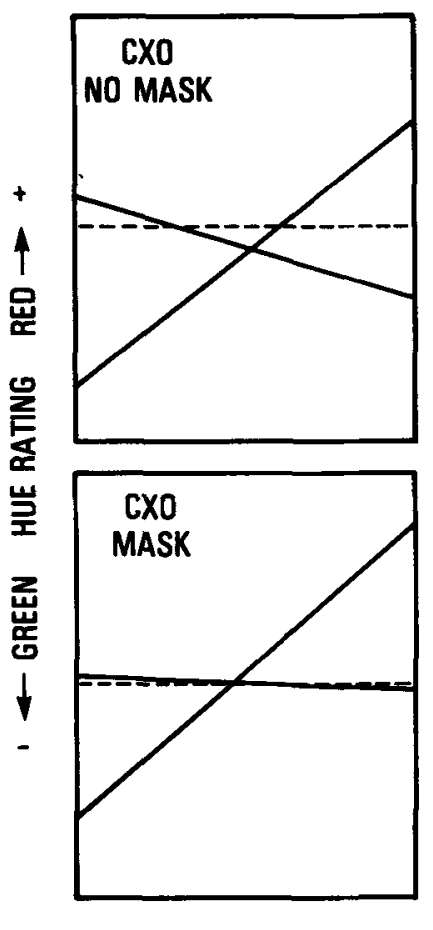

$-$
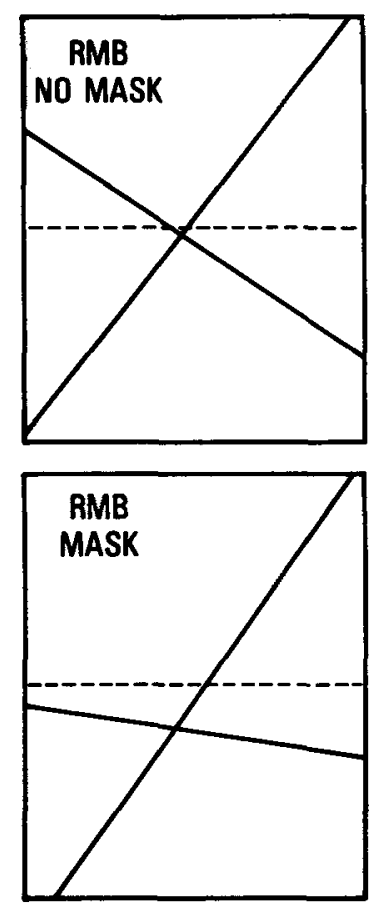

FIELD DIFFERENCE
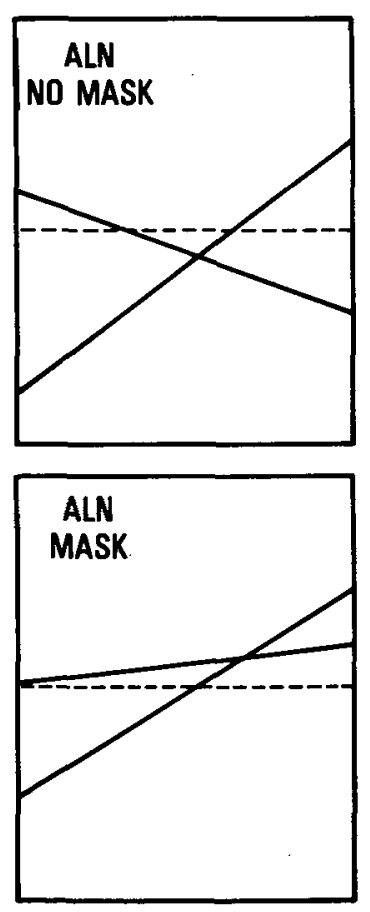

$+$

Figure 3. At the top of the figure are shown the regression lines for individual subjects, plotted without the data points, for Condition A (no-mask), which produced the mean data of Figure 2. At the bottom are plotted data for Condition B (with mask), for which mean data are given in Figure 4. The Pearson produce-moment coefficients of correlation of the data to these regression lines is as follows:

\begin{tabular}{lcc} 
& \multicolumn{2}{c}{ Without Mask } \\
Subject & Direct & Induced \\
C.X.O. & .94 & -.84 \\
R.M.B. & .98 & -.99 \\
A.L.N. & .81 & -.62
\end{tabular}

\begin{tabular}{cc}
\multicolumn{2}{c}{ With Mask } \\
Direct & Induced \\
.90 & -.15 \\
.98 & -.75 \\
.81 & .28
\end{tabular}

centrically arranged into spatially antagonistic and spectrally opponent regions, send afferent signals for processing in the cortex. As noted earlier, the singleopponent cells found in the lateral geniculate of the rhesus cannot account for induction. Michael has shown that the double-opponent cells in the cortex synapse with the single-opponent cells of the lateral geniculate, and he proposes that inputs from two cone systems converge to build double-opponent units at the cortical level. Valberg and Seim (1983) have also postulated a physiological model of chromatic induction based on Michael's data. Lateral excitation in the double-opponent cells may therefore account for the mechanism of color induction for flashed stimuli, where eye movements are prevented that otherwise could allow successive color contrast for conditions of steady viewing.

The conditions of our experiment were restricted to: (1) low photopic luminances, (2) small inducing and test fields that were equal in area and slightly separated, (3) fixation of the test field, (4) induction tested along the red-green dimension only, and (5) briefly flashed stimuli. For these conditions, the results reported above indicate that induction is mainly a nonretinal phenomenon, but they do not pinpoint whether the interaction between signals from inducing and test fields occurs before or after binocular combination in the visual cortex. If after, the percentage of dichoptic induction should be equal to that of monoptic induction. This was tested for one subject (R.M.B.) and found not to be so. The result is shown in Figure 5, where it can be seen that only a small amount of induction remains (roughly equivalent to that found for monoptic induction for this subject in the presence of the contralateral metacontrast mask).

Although it seems reasonable to conclude that the major component of chromatic induction has its basis in the monocular visual pathways beyond the retina, and very probably in the monocularly driven double-opponent cells, two unanswered questions remain. How do signals from the contralateral mask 


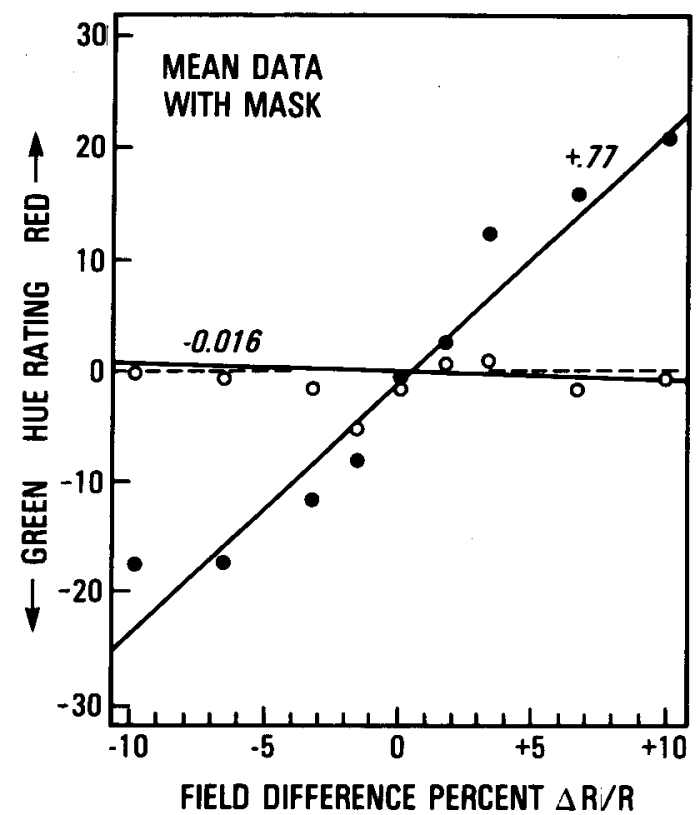

'Figure 4. Same as Figure 2, but for Condition B, in which the dichoptic metacontrast mask was used.

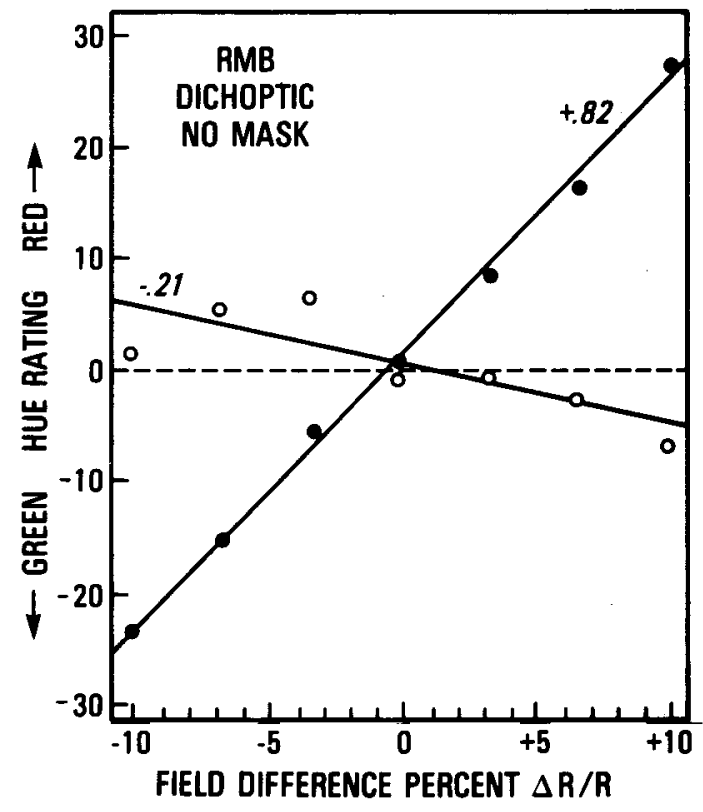

Figure 5. Individual data for R.M.B. from an experiment in which the inducing field was presented to the left eye, the test field to the right. No metacontrast mask was used (open and closed symbols are as in Figures 2 and 4).

manage to inhibit the chromatic induction normally mediated by monocularly driven double-opponent units? And, if monocular double-opponent cells mediate monoptic masking, what is the physiological basis of the much smaller amount of dichoptic induction that can also be demonstrated?
With respect to the first question, it should be pointed out that many cells classified as monocular by one set of criteria may nevertheless be influenced by appropriately chosen contralateral stimuli (Poggio \& Fischer, 1977). Thus, an electrophysiological test might show that Michael's double-opponent cells can be inhibited by contralateral flashes for conditions that produce metacontrast in humans. Where dichoptic induction is concerned, more data are needed to confirm that it reliably occurs for short flashes. If so, one would need to postulate some kind of activity in the brain that depends on binocular input in order to explain it. Our major conclusion is that chromatic induction in human subjects, at least for the conditions of our experiment, is largely and perhaps exclusively mediated by cells in the monocular visual pathways of the brain. Reasonable and likely candidates would seem to be the monocularly driven double-opponent units known to exist in the primate visual cortex.

\section{REFERENCES}

AlPERn, M. (1953). Metacontrast. Journal of the Optical Society of America, 43, 648-567.

AlPE RN, M. (1954). The effect of luminance of the contrast inducing flashes on the spatial range of metacontrast. American Journal of Optometry, 31, 363-369.

ALPERN, M. (1964). Relation between brightness and color contrast. Journal of the Optical Society of America, 54, 1491-1492.

Boynton, R. M., \& NAGY, A. L. (1981, December). The La Jolla analytic colorimeter: Optics, calibrations, procedures, and control experiments (CHIP Report No. 109). La Jolla University of California at San Diego, Center for Human Information Processing.

Boynton, R. M., \& NAGY, A. L. (1982). La Jolla analytic colorimeter. Journal of the Optical Society of America, 72, 666-667.

DeVAlois, R. L., \& Walraven, J. (1967). Monocular and binocular aftereffects of chromatic adaptation. Science, 155, 463-465.

Geschwind, N., \& Segal, J. R. (1960). Colors of all hues from binocular mixing of two colors. Science, 131, 608.

Graham, C. H., \& Brown, J. L. (1965). Color contrast and color appearance: Brightness constancy and color constancy. In C. H. Graham (Ed.), Vision and visual perception. New York: Wiley.

HeLmholtz, H. voN. (1924). Helmholtz's physiological optics (J. P. C. Southall, Trans.). Rochester, NY: Optical Society of America. (Original work published 1866).

Hering, E. (1890). Beitrag zur Lehre vom Simultankontrast. Zeitschrift für Psychologie, 1, 18-28.

Hurvich, L. M. (1981). Color vision. Sunderland, MA: Sinauer.

JAmeson, D., \& Hunvich, L. M. (1961). Opponent chromatic induction: Experimental evaluation and theoretical account. Journal of the Optical Society of America, 51, 46-53.

JAMEsON, D., \& HuRvich, L. M. (1964). Theory of brightness and color contrast in human vision. Vision Research, 4, 135-154.

Kinney, J. A. S. (1962). Factors affecting induced color. Vision Research, 2, 503-525.

KinNey, J. A. S. (1967). Color induction using asynchronous flashes. Vision Research, 7, 299-318.

Lefton, L. A. (1973). Metacontrast: A review. Perception \& Psychophysics, 13, 161-171.

MichaEL, C. R. (1978a). Color-sensitive complex cells in monkey striate cortex. Journal of Neurophysiology, 41, 1250-1266.

MichaEt, C. R. (1978b). Color vision mechanisms in monkey 
striate cortex: Dual-opponent cells with concentric receptive fields. Journal of Neurophysiology, 41, 572-588.

MichaEl, C. R. (1978c). Color vision mechanisms in monkey striate cortex: Simple cells with dual opponent-color receptive fields. Journal of Neurophysiology, 41, 1233-1249.

Mollon, J., \& Sharpe; T. (1983) (Eds.). Physiology and psychophysics. London: Academic Press.

Pargons, J. H. (1924). An introduction to the study of colour vision (2nd ed). Cambridge: Cambridge University Press.

Poggio, G. F., \& Fischer, B. (1977). Binocular interaction and depth sensitivity in striate and prestriate cortex of behaving rhesus monkey. Journal of Neurophysiology, 40, 1392-1405.

Smith, V. C., \& Poxorny, J. (1975). Spectral sensitivity of the foveal cone photopigments between 400 and $500 \mathrm{~nm}$. Vision Research, 15, 161-171.

UTtal, W. R. (1981). A taxonomy of visual processes. Hillsdale, NJ: Erlbaum.

Valaerg, A., \& Seim, T. (1983). Chromatic induction: Responses of neurophysiological double opponent units? Biological Cybernetics, 46, 149-158.
Walraven, J. (1981). Chromatic induction: Psychophysical studies on signal processing in human colour vision. Unpublished thesis, Institute for Perception, Soesterberg, The Netherlands.

Werner, H. (1940). Studies on contour: Strobostereoscopic phenomena. American Journal of Psychology, 53, 418-422.

Wiesel, T. N., \& HuBel, D. H. (1966). Spatial and chromatic interactions in the lateral geniculate body of the rhesus monkey. Journal of Neurophysiology, 29, $1115-1156$.

Wooten, B. R. (1970). The effects of simultaneous and successive chromatic contrast on spectral hue. Unpublished doctoral dissertation, Brown University, Providence, RI.

YeLLOTT, J. I., JR., \& WANDELL, B. A. (1976). Color properties of the contrast flash effect: Monoptic vs. dichoptic comparisons. Vision Research, 16, 1275-1280.

(Manuscript received December 6, 1983; accepted for publication February $18,1984$. 